\title{
Clinical investigation of an infrared digital scanning laser ophthalmoscope
}

\author{
A Manivannan, J N P Kirkpatrick, P F Sharp, J V Forrester
}

\begin{abstract}
An infrared scanning laser ophthalmoscope (SLO) has been custom built in order to investigate the application of confocal and indirect mode SLO imaging to patients with fundus disease. Infrared light is reflected from the fundus to a greater extent than visible light permitting lower illumination power and, as it penetrates the retinal pigment epithelium, choroidal structures can be readily imaged. Furthermore, as conventional infrared illumination and detection systems are not well suited to ophthalmoscopy, this area is underdeveloped as a potential source of useful clinical data. Confocal, direct and indirect imaging modes have been used to image fundi of normal volunteers and patients with fundus disease. In comparison with conventional fundus photography confocal infrared SLO imaging improves visualisation of choroidal vasculature, retinal pigment epithelial abnormalities, laser photocoagulation scars, and optic disc pores in the lamina cribrosa. Direct infrared SLO imaging enables fundus visualisation through nuclear lens opacities. Furthermore, indirect mode imaging enhances significantly the appearance of macular drusen. The potential clinical benefit of these observations is discussed.
\end{abstract}

(Brf Ophthalmol 1994; 78: 84-90)

Conventional fundus imaging, either by direct or indirect ophthalmoscopy, has a number of shortcomings. The optics of the eye dictate that both the illuminating light and the reflected light pass through the pupil. In direct ophthalmoscopy a mirror separates the illuminating and the reflected light and a direct one to one image of the patient's fundus is projected onto the observer's retina. While it remains the simplest method of funduscopy, its clinical value is reduced by the small field of view, poor image quality in the presence of media opacity, and the need for correction of ametropia. A wide field of illumination and a clear view of the retinal periphery is achieved by indirect ophthalmoscopy. The illuminating beam passes through the periphery of the pupil while the reflected light emerges through the centre of the pupil. As a result, however, it is necessary to dilate the pupil and use high levels of light, ${ }^{1}$ which may be uncomfortable for patients. Monochromatic ophthalmoscopy and fundus photography have been used to enhance visualisation of certain fundus structures by improving contrast and optical quality. ${ }^{2}$

Confocal scanning laser ophthalmology ${ }^{3}$ is a new method available to the ophthalmologist for the acquisition of fundus images. In the scanning laser ophthalmoscope (SLO) the entrance and exit pupils are inverted so that a narrow $1 \mathrm{~mm}$ beam of laser traverses the optical axis to a single $10 \mu \mathrm{m}$ point on the fundus. ${ }^{4}$ The portion of the reflected light which passes through the large exit pupil is detected. This creates a much more efficient system and permits lower intensity illumination with resulting comfort for the patient. A fundus image is generated by scanning the retina in a raster fashion and the reflected light is recorded by a detector linked to a monitor. The laser used may have a wavelength in any part of the visible or near infrared spectrum and will result in a monochromatic image of the fundus. Thus chromatic aberration is avoided.

An SLO based on these principles has recently been constructed at Aberdeen. ${ }^{5}$ In this paper the clinical potential that this technique offers is explored. While there is potentially a wide choice of wavelengths for the illuminating beam, initially emphasis has been placed on the use of infrared, for a number of reasons. Firstly, compared with visible light, infrared has a higher reflectivity from the human fundus thereby permitting lower illuminating power. ${ }^{6}$ Secondly, infrared light penetrates deeper into the fundus and should permit imaging of the choroid as well as the retina. Thirdly, an SLO provides clearer images than if infrared illumination were to be used in conventional fundus photography. ${ }^{8}$ In conventional fundus photography not only is light from the electronic flash polychromatic, thereby inducing aberration, but also it does not give a true infrared retinal image, as the recording film is partially sensitive to visible light. ${ }^{9}$

In the Aberdeen system reflected laser light is detected by a photodiode and directly digitised by a computer. This avoids the need for photographic or video recording with their attendant image distortion, while giving real time imaging, frame grabbing, and access to sophisticated image processing.

A further feature of the SLO is that it allows tomographic imaging of the fundus using a confocal aperture. The term confocal refers to the fact that detection takes place at, or through, a focal point which is conjugate to the focus of the 
illumination spot on the retina. ${ }^{3}$ Any light scattered from layers or points other than the point of illumination is blocked by a confocal aperture whose position thus determines the layer from which the reflected light is collected. As the diameter of the confocal aperture is reduced, then the depth of focus of the imaged layer also becomes smaller. Theoretically an aperture size of $10 \mu \mathrm{m}$ will result in a depth of focus of $100 \mu \mathrm{m}$ at the retina ${ }^{5}$ but only at the expense of a significant reduction in image intensity. In the present study aperture sizes as small as $200 \mu \mathrm{m}$ have been employed.

\section{Methods}

Patients attending the eye clinic, Aberdeen Royal Infirmary were selected for SLO imaging using the system previously described. ${ }^{5}$ These patients had a wide variety of ocular pathology. Ethics committee approval for the study was granted and informed consent was obtained. Most images were acquired using the near infrared laser in the confocal mode and compared with conventional fundus photography. Where indicated fundus fluorescein angiography and SLO imaging using a visible red laser $(670 \mathrm{~nm})$ were also undertaken. Using a central stop in the detection system allows detection of scattered light only and creates an image known as the indirect image. ${ }^{3}$ This method was used in the detection of drusen.

\section{Results}

A series of illustrative cases are presented.

\section{SLO IMAGING AT 670 AND 830 NM WAVELENGTHS} Case 1

Figure 1 shows images of the retina taken from a 25 -year-old healthy volunteer. Figure 1A shows the confocal image of the retina using a visible wavelength $(670 \mathrm{~nm})$. The fovea shows as a dark spot (arrow). The arteries (A) and veins (V) are well differentiated; the veins appear darker than the arteries as the deoxygenated blood absorbs the light at $670 \mathrm{~nm}$ to a greater extent than the oxygenated blood. ${ }^{10}$

Figure 1B shows a confocal image of the same subject taken at the retinal layer using the near infrared laser of $830 \mathrm{~nm}$ wavelength. The optic disc is well defined. The optic cup shows up as a bright area inside the optic disc. The arteries and veins are dark and have high contrast with respect to the retina. Variations in pigmentation at the retinal pigment epithelium show as dark patches.

The use of a confocal aperture in the detection system permits the SLO to 'focus' on layers deep to the retina. In Figure $1 \mathrm{C}$ the normal fundus has been imaged using $830 \mathrm{~nm}$ wavelength and a small confocal aperture $(200 \mu \mathrm{m})$. Small retinal vessels seen in Figure 1B have disappeared and the deeper choroidal vessels are evident (arrows). This technique is employed in some of the following images. It should be noted that confocal imaging at deep retinal levels using visible light $(670 \mathrm{~nm})$ yields a featureless image due to the poor penetrance of this wavelength.
Case 2

A 30-year-old volunteer with normal visual acuity was imaged at 670 and $830 \mathrm{~nm}$ using the SLO. Figure $2 \mathrm{~A}$ shows the colour fundus photograph of the left eye. In the upper macula there is an area of retinal pigment epithelial hyperplasia which shows as an area of pallor with a hyperpigmented border. Confocal imaging at $670 \mathrm{~nm}$ shows this region as an area of minimally increased intensity (Fig 2B). Whereas the image taken using $830 \mathrm{~nm}$ shows a highly reflective area through which the choroidal vasculature is just visible (Fig 2C).

\section{CHORIORETINAL SCARS FROM}

PHOTOCOAGULATION BURNS

Case 1

A 50-year-old male patient who had undergone
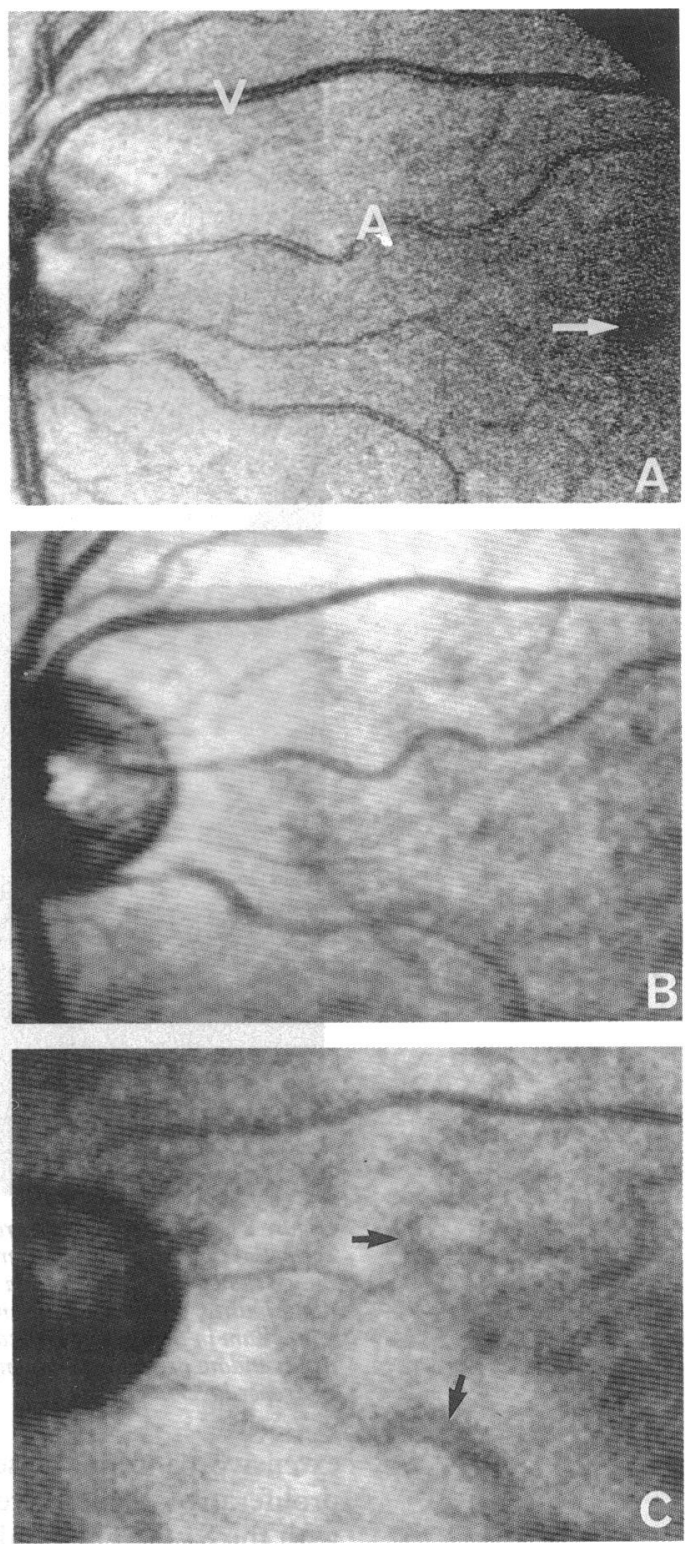

Figure 1 (A) Confocal image of the normal fundus at $670 \mathrm{~nm}$. Arteries $(A)$ appear lighter than veins $(V)$ due to different reflectance of oxygenated and deoxygenated haemoglobin. The fovea appears as a dark area (arrow). (B) Near infrared $830 \mathrm{~nm}$ confocal image. Arteries and veins appear dark. Note the highly reflective area of the optic cup within the darker rim of the optic disc. (C) Deep confocal image of macula at $830 \mathrm{~nm}$. Small retinal vessels disappear and choroidal vessels (arrow) become more obvious. 

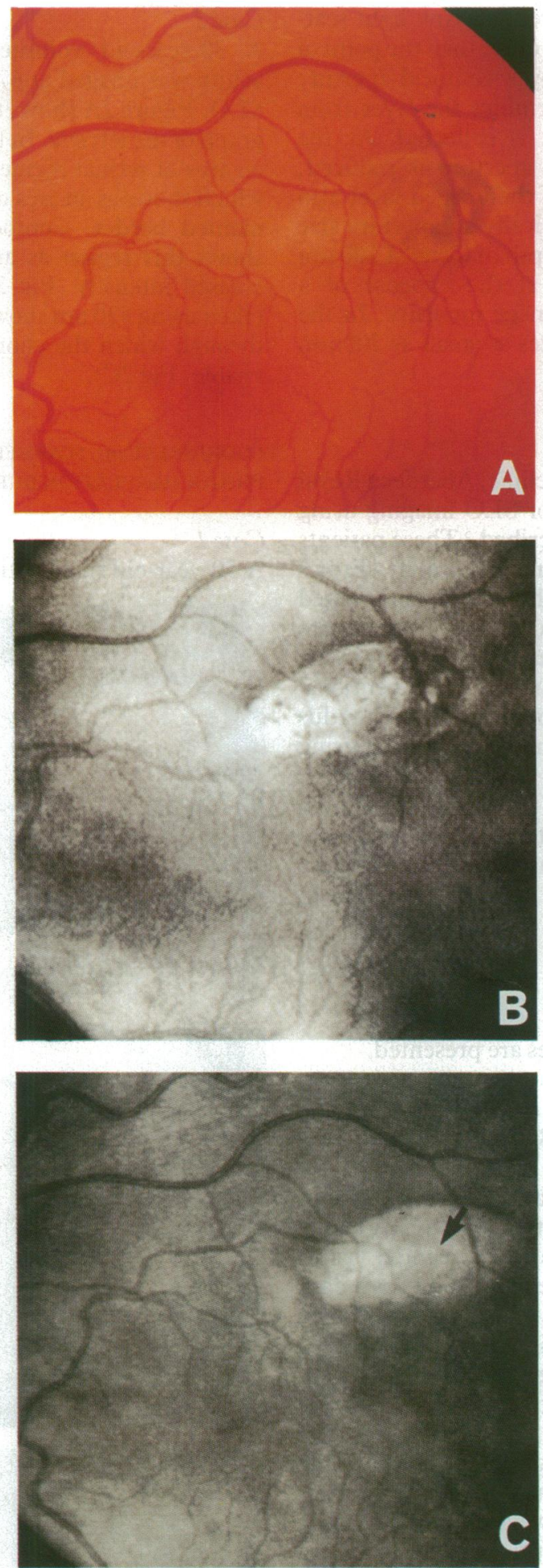

Figure 2 (A) Colour photograph showing pigment epithelial abnormality in upper macula. (B) $670 \mathrm{~nm}$ confocal image shows brighter reflection from the pigment epithelial abnormality. (C) In the $830 \mathrm{~nm}$ confocal image the pigment epithelium in the area of the scar is highly reflective and acts as a window permitting imaging of choroidal vessels (arrow) in deeper layers.

extensive panretinal laser photocoagulation for proliferative diabetic retinopathy was imaged with the SLO. The fundus and the SLO images of the left eye of the patient are shown in Figure 3. The photocoagulated areas (arrows) appear as yellowish red patches with diffuse edges in the fundus photograph (Fig 3A). The contrast of these areas with the neighbouring tissues is higher for the SLO image (Fig 3B) than that of the fundus camera. The reflectance characteris-
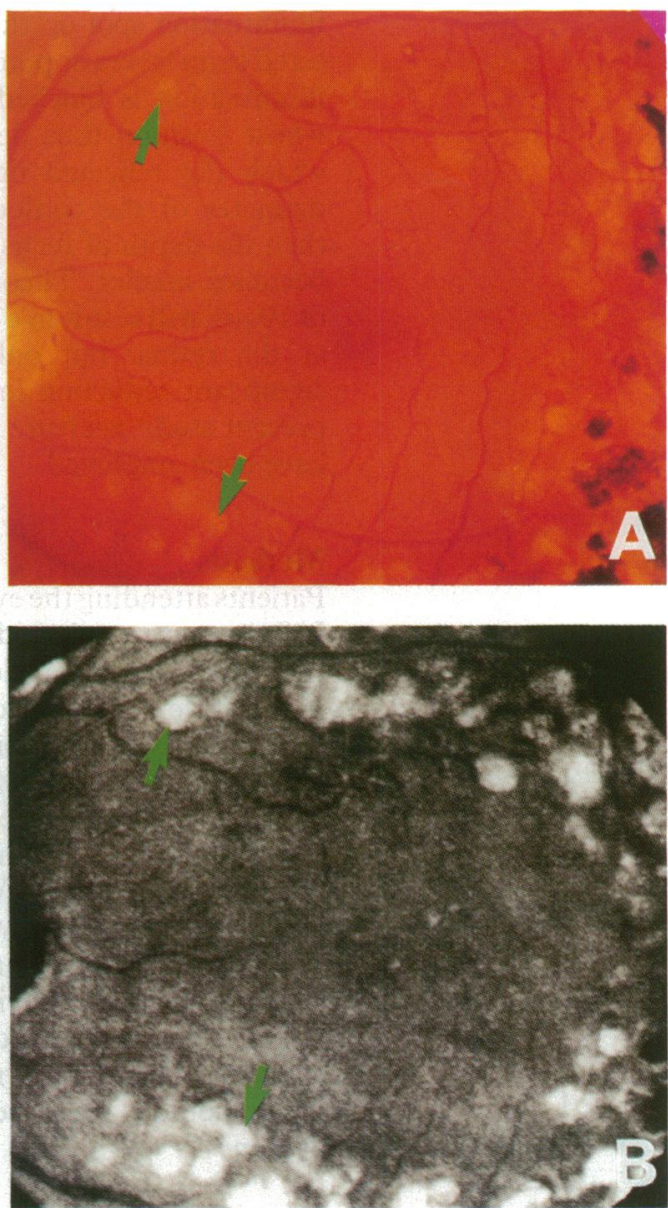

Figure 3 (A) Fundus photograph of a patient with photocoagulation scars after panretinal ablation. (B) Same area imaged with the $830 \mathrm{~nm}$ confocal scanning laser ophthalmoscope. Note that comparable laser burns (arrows) have higher reflectance in infrared light.

tics of the sclera with near infrared illumination are unknown and the sclera may be highly reflective at these wavelengths.

Case 2

A 76-year-old woman with a subretinal neovascular membrane treated by laser photocoagulation 2 weeks earlier was imaged with the confocal SLO. Figure 4 shows a colour photograph and the SLO image taken at the same time. The residual subretinal fluid causes slight pallor of the retina in the colour photograph and this shows as an edge of high contrast on the SLO image (arrowed). An area of high signal corresponds to the site of laser photocoagulation of the neovascular complex which is less visible on the colour photograph.

\section{IMAGING THROUGH LENS OPACITIES}

A 76-year-old female patient with severe nuclear cataract was imaged with the SLO. The fundus image of the patient is shown in Figure 5A. The SLO image of the patient shown in Figure 5B shows the retina in more detail. Retinal vessels, optic disc, and the macula are clearly visualised.

OPTIC DISC AND LAMINA CRIBROSA IMAGING A patient with posterior uveitis and peripapillary 
Figure 4 (A) Colour photograph of the left macula showing an area of retinal pallor (arrowed) corresponding to the site of subretinal fluid in a patient with subretinal neovascular membrane. The neovascular membrane has a grey

ippearance at the centre of the area. (B) Confocal scanning laser

ophthalmoscopic image of the same eye at $830 \mathrm{~nm}$. The area with residual subretinal fluid has a high contrast edge (arrowed) and the central neovascular membrane appears highly reflective due to laser photocoagulation.
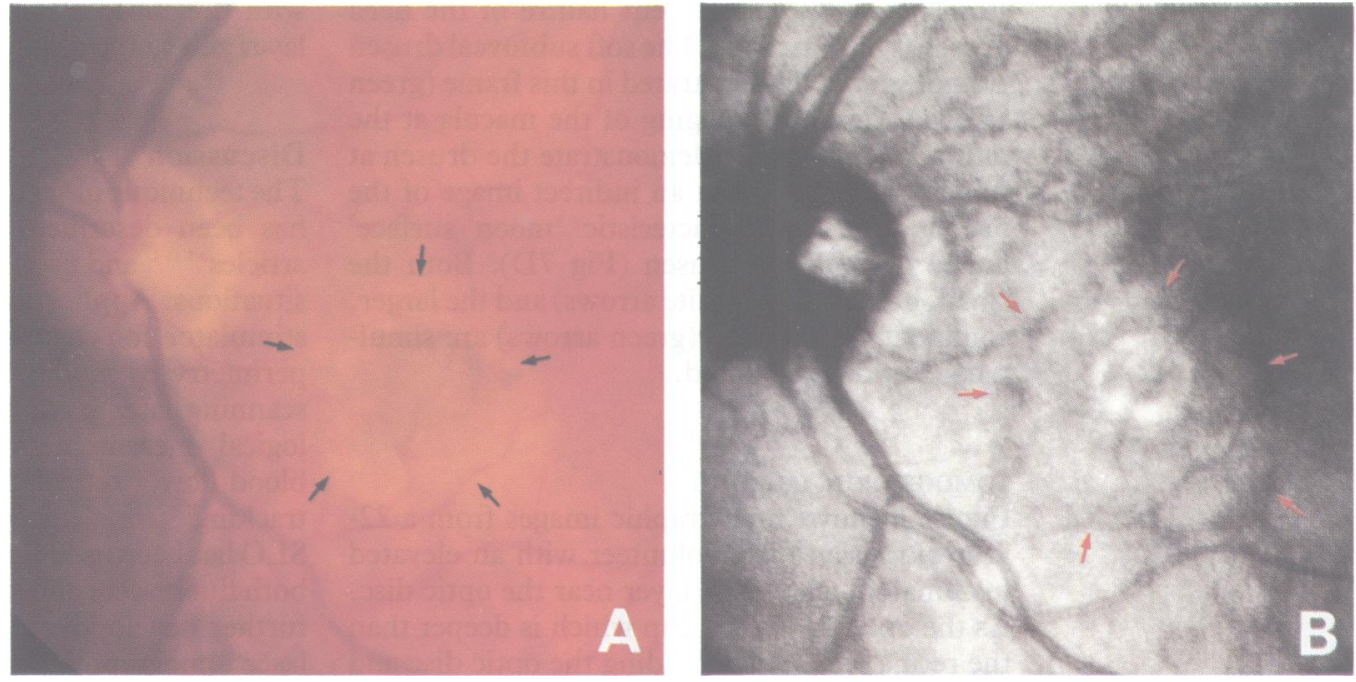

atrophy underwent SLO imaging concentrating on the optic disc. Figures $6 \mathrm{~A}$ and $6 \mathrm{~B}$ show the corresponding fundus photographic and SLO images. In the fundus photograph the disc shows signs of pallor, peripapillary atrophy, and vascular attenuation but no further detail is evident. The SLO image demonstrates some of the pores in the lamina cribrosa clearly with a dark surrounding appearance of the optic disc.
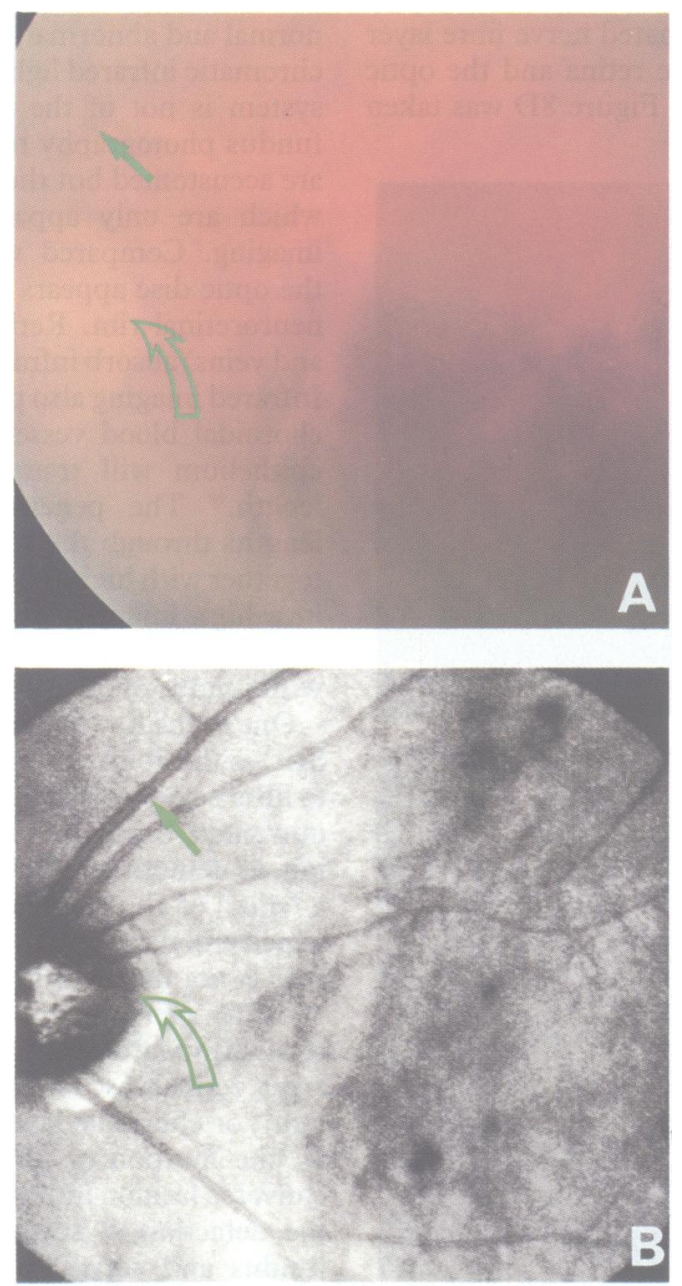

Figure 5 (A) Colour photograph showing poor fundus detail in a patient with dense nuclear sclerotic cataract. The optic disc (open arrow) and vessels (closed arrow) are barely visible. (B) The $830 \mathrm{~nm}$ scanning laser ophthalmoscope image shows fundus detail with greater clarity. The optic disc (open arrow) and vessels (closed arrow) are clearly shown.

\section{DRUSEN IMAGING}

A 73-year-old patient with macular drusen was imaged using $830 \mathrm{~nm}$ confocal and indirect imaging modes. Figure $7 \mathrm{~A}$ shows the fundus photograph of the left eye in which there are multiple drusen throughout the macula (white arrows). These are more confluent and 'softer' at the fovea (green arrows). Figure 7B shows a late fluorescein angiographic frame which demon-
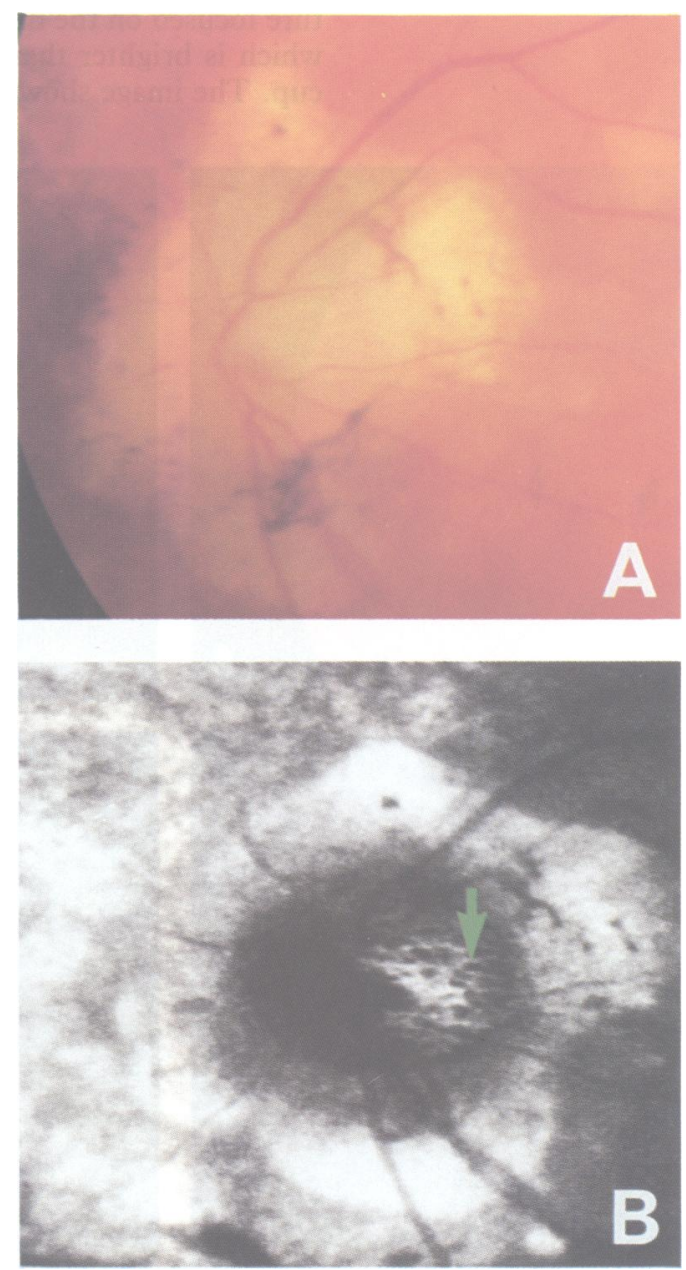

Figure 6 (A) Colour photograph of a patient with birdshot choroidoretinopathy and peripapillary atrophy. $(B)$ The near infrared confocal scanning laser ophthalmoscope image demonstrates greater contrast between optic disc pores (arrow) and the surrounding lamina cribrosa. 
strates the hyperfluorescent nature of the hard drusen (white arrows). The soft subfoveal drusen are not so well demonstrated in this frame (green arrows). Confocal imaging of the macula at the retinal layer does not demonstrate the drusen at this layer (Fig 7C) but an indirect image of the macula gives a characteristic 'moon surface' image of multiple drusen (Fig 7D). Both the small, hard drusen (white arrows) and the larger, confluent, soft drusen (green arrows) are simultaneously demonstrated.

\section{TOMOGRAPHIC IMAGING}

Figure 8 shows tomographic images from a 22year-old Asian male volunteer with an elevated myelinated nerve fibre layer near the optic disc. As the optic disc has a cup which is deeper than the retina, this area including the optic disc and the myelinated nerve fibre layer forms a structure containing a peak and a trough, which is suitable for tomographic study. The fundus image of the volunteer is shown in Figure 8A. The SLO images were captured with the infrared laser using the $200 \mu \mathrm{m}$ confocal aperture. The image shown in Figure 8B was taken with the confocal aperture focused on the retina and so the optic cup and the myelinated nerve fibre layer have almost the same brightness. The image shown in Figure $8 \mathrm{C}$ was captured with the confocal aperture focused on the myelinated nerve fibre layer which is brighter than the retina and the optic cup. The image shown in Figure 8D was taken
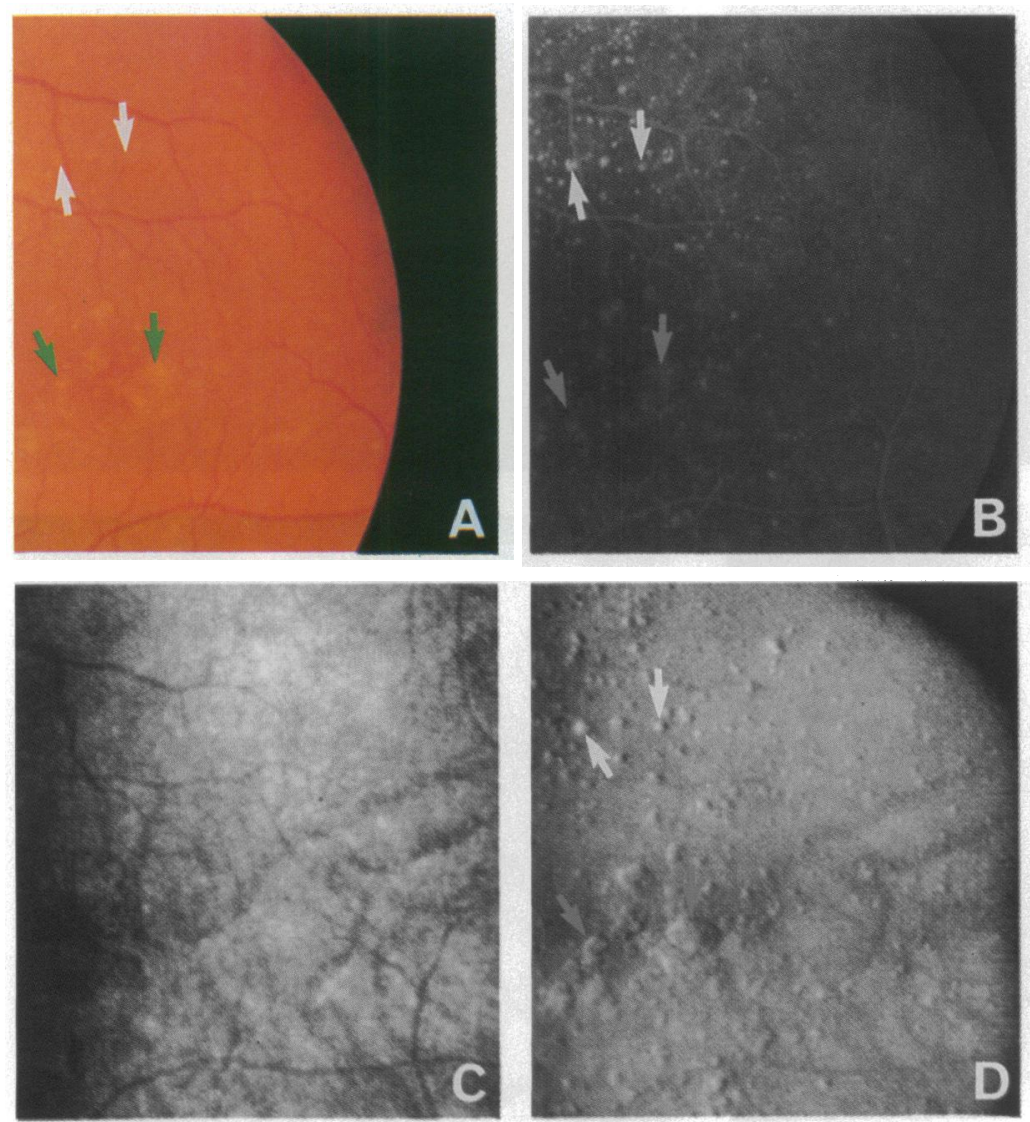

Figure 7 (A) Colour photograph of a patient with macular drusen. Hard drusen are marked with white arrows and the soft drusen with green arrows. $(B)$ Fluorescein angiographic frame in late venous phase demonstrates multiple hard (white arrows) and soft (green arrows) drusen. (C) Confocal $830 \mathrm{~nm}$ view at the retinal surface shows no abnormality. (D) Near infrared indirect scanning laser ophthalmoscope image confirms the presence of hard (white arrows) and confluent soft (green arrows) drusen with elevated appearance. with the confocal aperture focused at a deeper layer and so the optic cup looks bright.

\section{Discussion}

The technique of scanning laser ophthalmoscopy has been described in a number of previous articles $^{3511}$ and used in a wide variety of clinical situations. Applications of the SLO as a retinal stimulator for electrophysiological tests and in perimetry by modulation of the intensity of the scanning laser have been described. ${ }^{1314}$ Physiological measurements of perifoveal capillary blood flow, ${ }^{15}$ retinal densitometry, ${ }^{16}$ and fundus tracking ${ }^{17}$ using the SLO have been made. The SLO has been used for fundus angiography using both fluorescein and indocyanine green dyes. ${ }^{18} \mathrm{~A}$ further development has been that of scanning laser tomography which uses a confocal SLO to take serial images at different depths into the fundus and may give three dimensional information useful in the monitoring of glaucoma patients. ${ }^{19}$ The most frequent use of commercial SLOs, however, has been to provide high quality fluorescein angiograms with the advantage of direct access to the images and obviating the need for photographic processing.

The images obtained using the present SLO were taken without fluorescence. These demonstrate the appearance of fundus structures, both normal and abnormal, when viewed with monochromatic infrared light. Image clarity using this system is not of the standard of conventional fundus photography to which ophthalmologists are accustomed but there are significant features which are only apparent with infrared SLO imaging. Compared with visible wavelengths, the optic disc appears darker particularly at the neuroretinal rim. Retinal vessels (both arteries and veins) absorb infrared light and appear dark. Infrared imaging also permits visualisation of the choroidal blood vessels as the retinal pigment epithelium will transmit light of this wavelength. ${ }^{20}$ The penetration of infrared wavelengths through the retinal pigment epithelium together with high reflectivity of sclera will result in a high intensity appearance of chorioretinal scars, including laser burns, when compared with fundus photographs.

One of the key features of the versatility of the SLO is the ability to vary the imaging technique to filter selectively the relevant information from unwanted background details. Confocal imaging, as demonstrated above, permits selection of a 'slice' of the fundus to be studied. All light which emerges from the eye towards the detector that is not emanating from the plane conjugate with the aperture will be discarded. It is thus a straightforward task to focus on the nerve fibre layer, retinal vasculature, retinal pigment epithelium, or choroidal layer individually depending on the location of the object of interest. In a converse fashion indirect mode imaging relies on the detection of scattered light only from the fundus and serves to highlight any structure which acts as an efficient source of light scatter. This appears to be the case with retinal drusen which have a striking appearance when imaged with the SLO using the indirect mode (Fig 8D). Drusen are refractile deposits on Bruch's mem- 

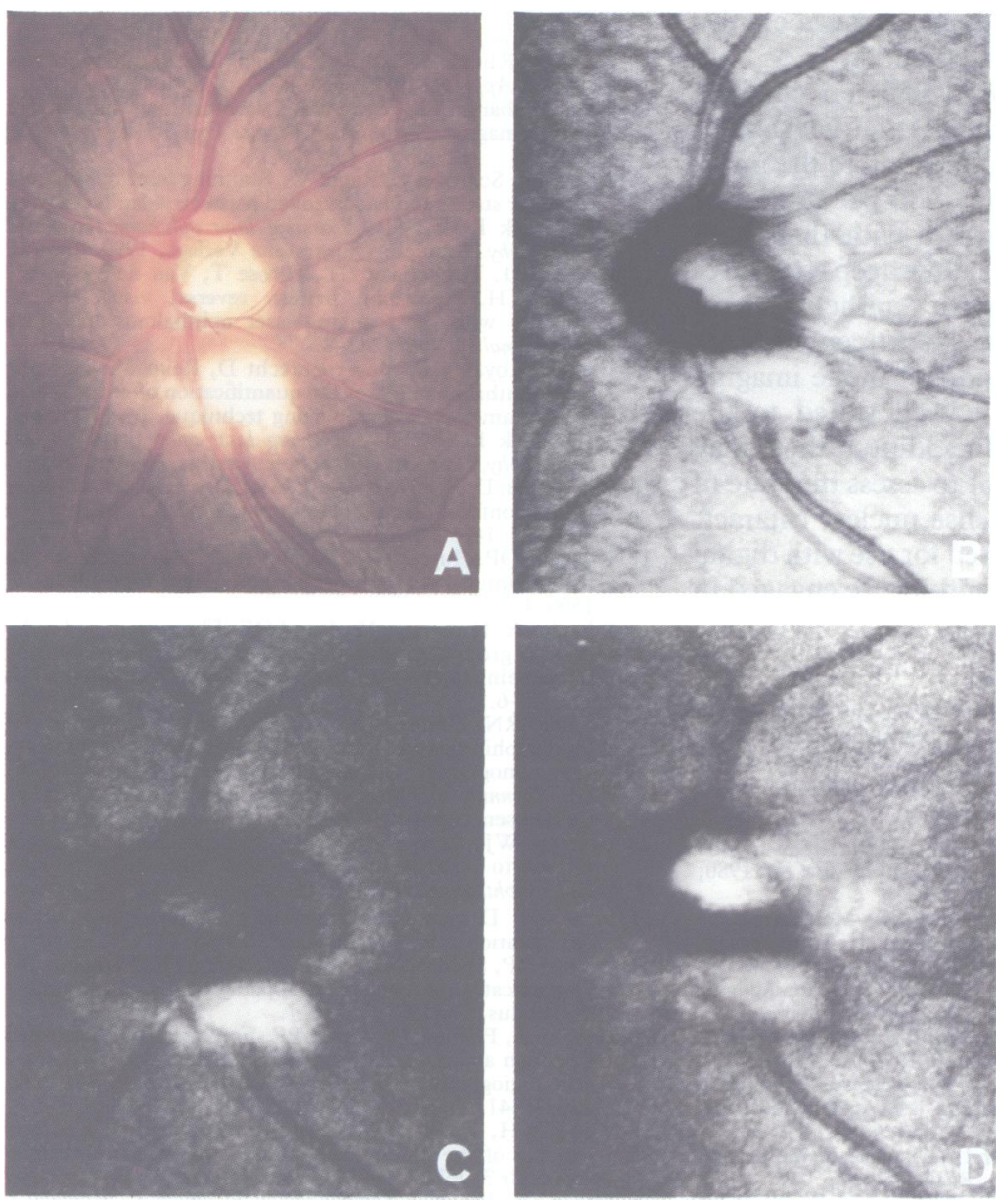

Figure 8 (A) Colour fundus photograph of eye with myelinated nerve fibres adjacent to optic disc. (B) A near infrared confocal image at the retinal level shows similar reflectance from the optic cup and myelinated nerve fibres. (C) Confocal image at the nerve fibre layer shows highly reflective nerve fibres compared with the surrounding retina and optic disc. (D) The deep near infrared confocal image at the level of the optic cup. The optic cup appears brighter than the surrounding structures.

brane often associated with age-related macular degeneration. They undergo a natural progression from small, so-called 'hard' drusen to larger, more confluent 'soft' drusen and may then disappear, leaving an area of retinal pigment epithelial atrophy, or they may promote the onset of exudative macular degeneration. ${ }^{21}$ Fluorescein angiography highlights drusen in greater numbers than photography, but the ideal time sequence for which the different types of drusen stain with fluorescein varies and thus prevents imaging of all types of drusen in a given angiographic frame. With the indirect mode, drusen appear as 'raised' structures (Fig 8D) which are easily visible and in numbers greater than those seen using fundus photography. These discrete lesions, with relatively clear edges, may thus lend themselves to image processing techniques similar to those which have already been applied to the diabetic macular changes such as exudates ${ }^{22}$ or microaneurysms. ${ }^{23}$ Automated counting and area determination might permit a greater understanding of the natural history of these lesions.

Fundus imaging in patients with dense cataract is difficult using conventional ophthalmoscopic techniques. In some patients, while there is a vague fundus view with an indirect ophthalmoscope it is difficult to record this adequately using a fundus camera. SLO infrared imaging in our patient gave a high degree of detail in comparison with the fundus photograph. This patient had a nuclear cataract and it is suggested that the monochromatic imaging technique, together with the highly collimated illuminating laser, may result in less scattering and allow a better focus on the retina. In addition, infrared wavelengths may have a greater transmission through this type of lens opacity. Scanning laser ophthalmoscopy may thus offer an advantage in the assessment of patients with cataract. This finding merits further investigation.

We have used the confocal images of a patient with myelinated nerve fibres close to the optic disc to demonstrate the tomographic capability of the SLO in vivo. These images were taken by adjusting the confocal aperture to image progressively deeper fundus layers. The high intensity reflection from the myelinated nerve fibres at the disc becomes successively reduced as deeper structures are imaged, while the intensity of reflected light from the optic cup changes in a paradoxical fashion. This suggests that the confocal imaging technique may serve as a guide to depth profile characteristics in the fundus.

In addition, the laser scanning tomographic technique has been developed to collect a series of confocal slices at narrow intervals which are then processed to yield data concerning optic disc configuration, optic cup size determination, and depth details. ${ }^{19}$ This instrument may be applicable to other fundus lesions which show variation in depth profile such as macular diseases and tumours.

The SLO can demonstrate detail of the lamina cribrosa more clearly than conventional imaging and this may serve as a further area of interest in monitoring optic nerve diseases. ${ }^{24}{ }^{25}$ Pore size and shape in the lamina cribrosa have been shown to be altered in glaucoma patients when compared with normal controls. ${ }^{26}$ Normal patients have dot-like pores whereas the pores of glaucoma patients show a slit-like appearance. However, not all patients have clinically visible pores in the lamina cribrosa as this structure is difficult to image because of lack of contrast in the surrounding optic disc. The SLO, when used in a confocal mode, permits acquisition of images which are 'focused' at the level of the lamina cribrosa and this, together with appropriate choice of wavelength, may permit greater differentiation of the lamina cribrosa from surrounding structures. The images presented here demonstrating lamina cribrosa pores are taken using a wider field of view than is necessary; it may be possible to further enhance image quality by altering the field size using optical magnification. ${ }^{25}$

SLO imaging in the patient who had undergone laser photocoagulation for a subretinal neovascular degeneration showed an interesting appearance of high intensity signal corresponding closely to the outline of the treated area. This photocoagulated area is not so easily demonstrated on the fundus photographic image. As in the previous patient who had photocoagulation, the confocal infrared image shows a high reflectance of light from areas with retinal pigment epithelial scars. It is thus easier to record which areas of the fundus have been photocoagulated and may be of use in clinical assessment of 
macular disease such as diabetic maculopathy and age-related macular degeneration.

In summary, the infrared $830 \mathrm{~nm}$ confocal SLO constructed in Aberdeen is an instrument which may have clinical application to the imaging of certain fundus features. The penetration of infrared wavelengths, together with their high reflectance from the sclera, allow deep structures such as the retinal pigment epithelium and choroid to be studied. This instrument also has the versatile function of indirect mode imaging which appears particularly well suited to the study of macular drusen. In addition, there may be a role for this instrument to assess the state of fundus in patients with dense nuclear cataract. The SLO is also ideally suited for use with digital image processing systems and we are engaged in further development of these.

This work is supported by grants from Wellcome Trust, Scotia Pharmaceuticals, and ACTR funds from Scottish Office Home and Health Department. We are grateful to Mr R Hutcheon, Mr D Eaton, and Mrs A Farrow for their photographic assistance.

1 Delori FC, Parker JS, Mainster MA. Light levels in fundus photography and fluorescein angiography. Vision Res 1980; 20: 1099-104.

2 Ducrey NM, Delori FC, Gragoudas ES. Monochromatic ophthalmoscopy and fundus photography. II. The pathological fundus. Arch Ophthalmol 1979, 97: 288-93.

3 Webb RH, Hughes GW, Delori FC. Confocal scanning laser ophthalmoscope. Appl Opt 1987; 26: 1492-9.

4 Webb RH, Hughes GW, Pomerantzeff O. Flying spot TV ophthalmoscope. Appl Opt 1980; 19: 1991-7.

5 Manivannan A, Sharp PF, Phillips RP, Forrester JV. Digital fundus imaging using a scanning laser ophthalmoscope. Physiol Meas 1993; 14: 43-56.

6 Delori FC, Pflibsen KP. Spectral reflectance of the human ocular fundus. Appl Opt 1989; 28: 1061-77.

7 Ham WT. Remarks of fundus reflectance. Vision Res 1975; 15: 1167-8.

8 Manivannan A. Digital fundus imaging with a scanning laser ophthalmoscope. PhD Thesis. University of Aberdeen, 1993.

9 Dallow RL. Colour infrared photography of the ocular fundus. Arch Ophthalmol 1974; 92: 254-8.
10 Behrendt T, Wilson L. Spectral reflectance photography of the retina. Am f Ophthalmol 1965; 59: 1079-88.

11 Plesch A, Klingbeil U, Bille J. Digital laser scanning fundus camera. Appl Opt 1987; 26: 1480-6.

12 Wynn-Williams GM, Crowe WE. Laser scanning: a method of retinal imaging. Australasian Physics Eng Sci Med 1986; 9 153-62.

13 Sturmer J, Schrodel C, Rappl W. Scanning laser ophthalmoscope for static fundus-controlled perimetry. In: Nasemann JE, Burk ROW, eds. Scanning laser ophthalmoscopy and JE, Burk ROW, eds. Scanning laser ophthalmoscopy and 14 Katsumi O, Timberlake GT, Hirose T, Van de Velde FJ Sakaue H. Recording pattern reversal visual evoked response with the scanning laser ophthalmoscope. Acto response with the scanning

15 Wolf S, Koyama T, Meyer-Ebrecht D, Reim M. Scanning laser ophthalmoscopy for the quantification of retinal blood flow parameters: a new imaging technique. In: Naseman JE, Burk ROW, eds. Scanning laser ophthalmoscopy and tomography. Munich: Quintessenz Verlags, 1990: 91-6.

16 Van Norren D, Van De Kratts J. Imaging retinal densitometry with a confocal scanning laser ophthalmoscope. Vision Res 1989; 29: 1825-30.

17 Wornson DP, Hughes GW, Webb RH. Fundus tracking with the scanning laser ophthalmoscope. Appl Opt 1987; 26: $1500-4$.

18 Scheider A, Nasemann JE, Lund OE. Fluorescein and indocyanine green angiography of central serous choroidopathy cyanine green angiography of central serous choroidopathy by scannin $50-6$.

19 Weinreb RN, Dreher AW. Reproducibility and accuracy of topographic measurements of the optic nerve head with the laser tomographic scanner. In: Nasemann JE, Burk ROW eds. Scanning laser ophthalmoscopy and tomography. Munich Quintessenz Verlags, 1990: 177-82.

20 Geeraets WJ, Berry ER. Ocular spectral characteristics as related to hazards from lasers and other light sources. Am F Ophthalmol 1968; 66: 15-20.

21 Sarks SH. Drusen and their relationship to senile macular degeneration. Aust f Ophthalmol 1980; 8: 117-30.

22 Phillips RP, Spencer T, Ross PGB, Sharp PF, Forrester JV. Quantification of diabetic retinopathy by digital imaging of the fundus. Eye 1991; 5: 130-7

23 Spencer T, Phillips RP, Sharp PF, Forrester JV. Automated detection and quantification of microaneurysms in fluorescein angiograms. Graefes Arch Clin Exp Ophthalmol 1992 230: $36-41$.

24 Woon WH, Fitzke FW, Bird AC, Marshall J. Confoca imaging of the fundus using a scanning laser ophthalmoscope. Br F Ophthalmol 1992; 76: 470-4.

25 Fitzke FW, Woon H, Timberlake G, Robinson L, Marshall J, Bird AC. Optical modifications to a scanning laser ophthalmoscope for high magnification, narrow optical section imaging. Laser and Light in Ophthalmology 1991; 4: 7-14.

26 Miller KM, Quigley HA. The clinical appearance of the lamina cribrosa as a function of the extent of glaucomatous optic cribrosa as a function of the extent of glaucomage
nerve damage. Ophthalmology 1988; 95: 135-8. 